\title{
The time-course of context modulation for underspecified meaning: an eye-movement study
}

\author{
Yao-Ying Lai ${ }^{\mathrm{a}, \mathrm{c}, *}$, David Braze ${ }^{\mathrm{b}}$, Maria Mercedes Piñango ${ }^{\mathrm{a}}$ \\ ${ }^{a}$ Department of Linguistics, Yale University, New Haven, CT, USA \\ ${ }^{b}$ Haskins Laboratories, New Haven, CT, USA \\ ${ }^{c}$ Section of Neuropsychology, National Rehabilitation Center for Persons with Disabilities, Tokorozawa, Japan
}

\begin{abstract}
Sentences such as "The pop singer began the album" are ambiguous between an agentive reading (The pop singer began recording/listening to/etc. the album) and a constitutive reading (The pop singer's song was contained in the first track of the album). The ambiguity is rooted in the meaning specification of a semantic class, the "aspectual verb class." This semantic verb class demands that its complement be construed as a structured individual along a dimension (e.g., spatial, informational, eventive...). In the case of "begin the album," the complement "the album" can be construed as a set of singing eventualities (a structured individual construed along the eventive dimension) or as a set of songs (a structured individual construed along the informational dimension). Previous work has shown that real-time sentence composition profile of these verbs is consistent with the implementation of two processes (a) exhaustive lexical-function retrieval and (b) construal of multiple dimension-specific structured individuals, leading to agentive and constitutive readings. Which dimension-specific structured individual is chosen ultimately depends on which dimension biases are present in the context. Understanding the real-time behavior of aspectual-verb composition under different contexts (agentive vs. constitutive) provides a glimpse into the time-course of context modulation during real-time sentence comprehension. Results from an eye-tracking study comparing agentive vs. constitutive readings of these sentences show not only the standard effect of the aspectual-verb composition, previously reported for the agentive readings, but also a comparable processing profile for the constitutive readings, a novel finding supporting the unified linguistic analysis and corresponding processing implementation of the two readings. Results also show that regardless of reading, the aspectual-verb composition effect is observable even after the complement has been retrieved, and interpretation has been disambiguated, thus indicating that the above two-step process is a nonnegotiable component of the interpretation of the verb+complement composition and therefore must take place before preceding context can serve as a sentence-external constraining force. Moreover, the sustained nature of the cost suggests that all possible dimension-specific structured individuals are being construed at least immediately after the complement has been retrieved, consistent with comprehenders' intuitions. This suggests that although not directly relevant for the interpretation at issue those meaning construals are not incompatible with the complement's conceptual representation, and therefore need not be "pruned." Overall, the pattern observed suggests a model of comprehension whereby context is allowed to modulate sentence interpretation once the fundamental lexico-semantic compositional processes of the sentence have taken place.
\end{abstract}

Keywords: semantic underspecification, sentence processing, semantic ambiguity, context, aspectual verbs, complement coercion

\section{Introduction}

The sentence comprehension system is known to make use of relevant contextual cues to determine the intended sentence meaning. When and under what conditions context modulates meaning composition as comprehension unfolds is less clear. Here we examine the time-course of context-sentence meaning interaction specifically in a case when sentence comprehension depends on meaning disambiguation rooted in lexical underspecification. That is the case of sentences with aspectual verbs such as begin, continue and finish, as in (1) below. In their transitive form, sentences with aspectual verbs

${ }^{*}$ Corresponding author

Email address: yaoyinglai@gmail . com (Yao-Ying Lai) 
give rise to multiple readings as in (1a-b). In such cases, the presence of the meaning ambiguity is not morpho-syntactically encoded (i.e. does not have a correspondingly ambiguous syntactic structure) yet it cannot be avoided.

(1) Dave Brubeck began this CD of jazz hits.

a. $\rightarrow$ Dave Brubeck began recording, etc.) a $C D$ of jazz hits. (agentive reading)

b. $\rightarrow$ Dave Brubeck's song was the first track on a CD containing jazz hits. (constitutive reading)

Example (1a) illustrates the "agentive reading." In this reading the subject referent is construed as an agent performing some activity involving the complement denotation. On the other hand, (1b) illustrates the "constitutive reading." In this reading the subject referent is construed as a subpart of an individual denoted by the complement. While the complement (this CD) is interpreted as a set of music playing-events in the agentive reading, in the constitutive reading it can be interpreted, say, as a physical entity (recorded songs on a plastic disk) or as a body of informational content (set of songs encoded in the plastic disk). In this view, the ambiguity observed in sentences with aspectual verbs is rooted to the multiple dimensions or perspectives along which the structure associated with the complement denotation can be construed-eventive, spatial, informational, etc.. This observation indicates that when retrieved, the complement's meaning need not be specified for dimension. This is what allows "this CD" to be interpreted along more than one such dimension/perspective (as an event, a physical entity, or informational content). The observation also indicates that this multiplicity in dimension percolates to the sentence level, leading to multiple readings which morphosyntactic cues alone cannot resolve. In the face of such sentence-level dimension ambiguity, comprehenders have to search for dimensiondisambiguating information in context in order to establish the intended interpretation of the sentence in question.

Whereas previous studies have shown that comprehension of sentences containing aspectual verbs (as compared to sentences containing psychological verbs, such as enjoy and love) engenders addi- tional processing cost and localizable cortical activity (DiNardo, 2015; Katsika et al., 2012; Lai et al., 2017a), the process by which context interacts with the comprehension process for the purposes of disambiguation is not well understood. ${ }^{1}$ Regarding the time-course of context effect during comprehension of underspecified meaning manifested by aspectualverb sentences, we consider three distinct possibilities: (i) immediate interaction indicated by absence of cost under biasing context, (ii) delayed interaction indicated by presence of cost under biasing context that disappears soon after the verb and the complement have been retrieved, and (iii) extrasentential interaction indicated by presence of cost under biasing context that remains well after the complement has been retrieved.

In the remainder of this section, we introduce the linguistic factors involved in the processing of aspectual-verb sentences (Section 1.1), address the role of context in the resolution of semantic ambiguity arising from the lexical semantics of aspectual verbs (Section 1.2) and present the details of the current study (Section 1.3).

\subsection{Hypothesis of processing sentences with aspec- tual verbs}

Our view of aspectual verbs as a lexico-semantic class is based on Piñango and Deo (2015)'s analysis. Specifically, they propose that aspectual verbs select for a structured individual as their complements. They define structured individual as an entity that can be construed as a one-dimensional path structure or axis, along some ontological dimension (e.g., spatial, informational, eventive, temporal, etc.). These dimensions can be thought of as arising from different perspectives on the verbal complement. For example, "a book" can be conceived as referring to a physical entity consisting of pages, binding, and covers, or a body of abstract informational content consisting of chapters or stories. Different dimensions may be independent of one another. For example, the first chapter of a book as informational content does not need to be printed on the first page of the book when book is construed as a physical entity. In this analysis, the mapping from the structured individual denoted by the complement to various dimensions is achieved by dimension-specific

\footnotetext{
${ }^{1}$ We note that prior to Katsika et al. (2012), all work on the comprehension of verbs like finish, begin did not distinguish the aspectual kind from other semantic verb classes that give rise to similar activity readings, including psychological such as enjoy, endure and others such as master, manage, based on the assumption that they all select for an event-denoting complement (e.g., Jackendoff, 1997; Husband et al., 2011; Frisson and McElree, 2008; McElree et al., 2001; Traxler et al., 2002, 2005; Pickering et al., 2005; Pustejovsky, 1995). Subsequent work has shown that such generalization is not correct. For sentences containing aspectual verbs specifically, there is also the possibility of a constitutive reading as in (1b) above and cases like "The editor's monologue begins the book," suggesting that the complement following these verbs need not denote an event, that instead it can select for an entity, and in contrast with sentences with psychological verb counterparts which crucially lack this second option. Accordingly, we focus on aspectual verbs in contrast to psychological verb counterparts because they contrast in terms of the presence of lexically-driven ambiguity that crucially demands relevant context to resolve and in this way allows us to examine in detail the question of time-course of context modulation.
} 
functions (e.g., $f_{\text {space }}, f_{\text {info }}, f_{\text {event }}, f_{\text {time }}$ ) lexically encoded in each verb of the class. Herein lies the source of the ambiguity: for any aspectual-verb sentence, the presence of the dimension-specific functions means that regardless of the complement it can be construed along all the possible dimensions along which it can be understood. Accordingly, achieving a unique interpretation necessitates appeal to the perceived intended dimension, that is reference to the context in which the sentence is uttered.

According to the Structured Individual Hypothesis (SIH, Lai et al., 2017a), semantic analysis of aspectual-verb sentences presented above is implemented over two sub-processes during real-time comprehension: (1) exhaustive retrieval of the aspectual verb's lexical functions and (2) subsequent resolution of dimension ambiguity. Previous evidence for this processing implementation comes from Lai et al.'s (2017a) self-paced reading and fMRI study. It shows that aspectual-verb sentence processing engenders (a) longer reading times as compared to control counterparts at the downstream of the complement head, and (b) temporally sequenced preferential recruitment of two cortical areas: left temporal-parietal area recruitment when the verb is encountered, associated with exhausted lexical retrieval, and the left inferior frontal gyrus (LIFG) after the complement head is encountered, associated with ambiguity resolution-a process which presupposes the construal and maintenance of structured individuals along their mutually exclusive dimension representations.

Figure 1 presents a visualization of the sources of processing cost as described by the SIH.

(2) Structured Individual Hypothesis

A. Exhaustive lexical retrieval: The processor retrieves all dimension functions encoded in the aspectual verb (e.g., $\left.f_{\text {space }}, f_{\text {info }}, f_{\text {event }}, f_{\text {time }}\right)$ when the verb is encountered.

B. Construal and maintenance of multiple dimension representations and ambiguity resolution: The composition of the verb's lexical functions and the structured individual conceptualized by the complement denotation necessitates the construal of multiple structured individuals along each of the dimensions available (e.g, spatial, informational, eventive, temporal) and the selection of one on the basis of context.

The time-course of context modulation in aspectual-verb sentence comprehension is particularly telling for two reasons: (1) the compositional processes at play are exclusively semantic and (2) disambiguation can only take place through semantic context. So close examination of this construction allows us to isolate those combinatorial properties of meaning comprehension from those involving morphological and syntactic composition. Before proceeding with our study, we present previous results on aspectual-verb sentence composition in connection to context modulation.

\subsection{Context modulation and aspectual-verb sen- tence composition: previous evidence}

Other studies have investigated context modulation during comprehension at least partly in connection to aspectual-verb comprehension. Traxler et al. (2005) found, via examining eye-movements, that introducing an activity with high frequency and high plausibility in context did not attenuate the processing cost in the subsequent aspectual-verb target sentence.

(3) Context: The student was reading in his office.

Target: After a while, he started a book about health care spending. (Traxler et al., 2005)

They also found that the cost was attenuated when the context sentence contained the full verb + complement construction, either same as in the target sentence (4a) or with the whole event structure explicitly mentioned (4b). Notably, the context sentence shows (a) > (b) in reading times (RTs); a difference that disappears in the subsequent target sentence, thus indicating the possibility of context modulation for the comprehension of aspectual-verb sentences.

(4) Context: The student (a) started /(b) read a book in his office.

Target: Before he (a) started / (b) read a book about opium trade, he checked his email.

Although Traxler et al.'s results are only partially informative due to the fact that the verbs used involved not only aspectual verbs but also psychological verbs (e.g., enjoy, prefer), along with other classes of verbs (e.g., manage, master), they already suggest that the immediacy of the time-course of context attenuation may be dependent on the strength of the contextual cue (the actual presentation of the same linguistic segment at issue being the strongest cue of all).

More recent evidence specific to aspectual-verb sentences already indicates that context cues do bias comprehenders to obtain the intended reading for 


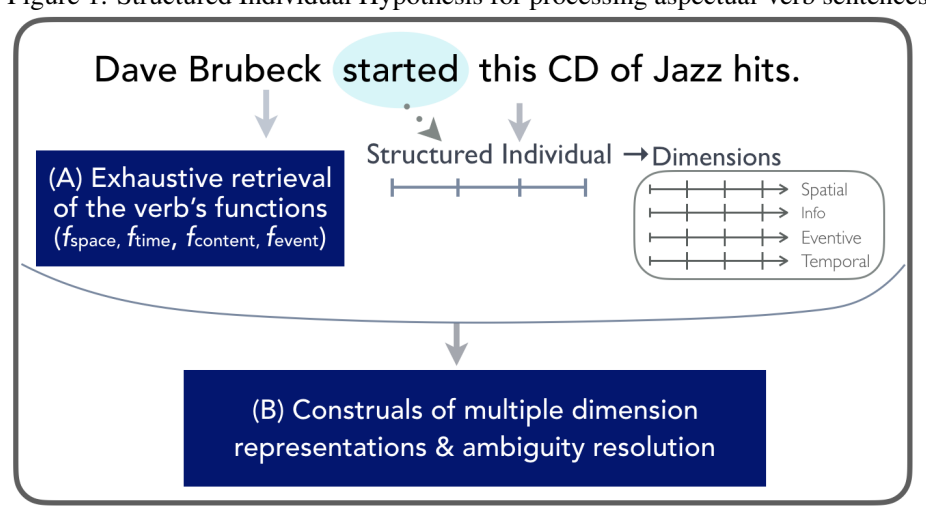

sentences like (1). Lai and Piñango (2017b, under review) report a questionnaire study whereby ambiguous aspectual-verb sentences such as "The lady began the novel." were preceded by biasing versus neutral context. They find that comprehenders arrive at an agentive reading of an aspectualverb sentence when the prior context sentence biases toward that reading by mentioning an activity in which the subject referent serves as an actor/agent. By the same token, comprehenders obtain a constitutive reading when an individual with salient subpart structure, which facilitates the constitutive reading, is mentioned in context. Furthermore, when the context remains neutral such that both readings are equally available, both agentive and constitutive readings are obtained with no significant difference in reading-selection rate. This offline study directly evidences that contextual information modulates the ultimate interpretation of aspectual-verb sentences.

This said, the timing of context modulation in real-time comprehension of sentences that involve underspecified meaning ambiguity remains a question. Uncovering this would reveal the fundamental internal articulation of the architecture of the sentence meaning composition subsystem and therefore of the interface between language composition and real-time cognitive processing. Meaning composition in such sentences, being exclusively semantic in nature, is the perfect window to observe this subsystem at work.

Coming back to possible roles of context, we note two distinct roles associated with (2B) in the processing of aspectual-verb sentences: context predetermines one specific interpretation thus suppressing the competing alternatives, or context privileges one interpretation without eliminating the alternatives. If context pre-determines an interpretation, the cost associated with construing multiple dimension-specific structured individuals will never arise, because only the corresponding function will be retrieved and only one structured individual will be construed to satisfy it. In this case, processing of an aspectual-verb sentence should be less costly when following a context which contains dimension-biasing information than following context which does not. Given Traxler et al.'s mixed results, we reason that the second possibility-context as privileger-is the more likely scenario. If context serves to privilege the favored dimension(s) from the set of already construed dimension-specific structured individuals without completely suppressing the alternatives, the impact of context modulation on aspectual-verb sentences would not emerge at least up to encountering the complement. This would predict cost for aspectual verb + complement composition regardless of context. That is, the question we address here is: when exactly during the unfolding of the sentence would the cost attenuation take place: right at the complement, downstream from the complement, or extra-sententially. Adjudicating between these possibilities is the objective of our study.

\subsection{The current study}

We investigate the time-course of context modulation in the comprehension of underspecified sentential meaning by examining the processing profile of ambiguous aspectual-verb sentences within biasing versus neutral contexts, via an eye-tracking experiment. We focus on two readings associated with aspectual-verb sentences - the agentive and constitutive readings as shown in (1). The ambiguity of these sentences is associated with the availability of two distinct dimension-functions, resulting in two thematic structures for the agentive and constitutive readings respectively, exemplified by (5).

(5) Dave Brubeck started this CD.

a. Agentive: <Agent, Theme> ex: Dave Brubeck started recording/listening to/burning/etc. this $C D$.

b. Constitutive: <Theme, Location> ex: Dave Brubeck's song was the first track on this $C D$. 
According to previous findings (e.g., Shapiro et al., 1989; Shetreet et al., 2016; Swinney, 1979), all argument structures of verbs are exhaustively retrieved. Therefore, we hypothesize that regardless of context, all dimension functions encoded in aspectual verbs will be retrieved, construing multiple dimension representations in composition with the complement. This is predicted to incur more processing cost in aspectual-verb composition as compared to the control counterparts.

Regarding the time-course of possible context modulation on the processing cost associated with underspecified meaning in real-time comprehension, we consider three plausible outcomes specifically.

(i) First, the cost associated with meaningunderspecified sentences is completely absent under a prior biasing context that resolves dimension ambiguity, indicating that the context is serving to predetermine the meaning. Given previous evidence, this is the least likely outcome but one that our experimental setup allows us to evaluate.

(ii) Second, the cost is present under biasing context yet it is eliminated upon or immediately after the complement is encountered, indicating that context serves to privilege one dimension-function over competing ones that were also retrieved and whose activation slowly decays.

(iii) Third, the cost is present under biasing context and remains even after the complement is retrieved, indicating that whatever privileging properties context has, it does not contribute to the decay of the alternative ones immediately. One reason for it would be that the alternatives are still compatible with the conceptual representation of the complement (e.g., "the book" can refer to the informational content or the physical pages contained in it).

\section{Methods}

\subsection{Materials}

Thirty sets of passages were created, each consisting of a context sentence followed by a target sentence, which contained either an aspectual verb or a control psychological verb (e.g., enjoy, love, see Footnote 1). Each aspectual-verb target sentence was paired with three types of context: (1) a context biasing towards the agentive reading along the eventive dimension (agentBiasingAspV), (2) a context biasing towards the constitutive reading along the informational or spatial dimension (constBiasing-AspV), or (3) a neutral context that is compatible with both the agentive and constitutive readings (Neutral-AspV). In the agentBiasing context, an action was mentioned to facilitate the agentive reading in which the subject is conceived of as an agent. In the constBiasing context, an entity referring to a structured individual was made salient while no action was mentioned. On the other hand, each control target sentence with psychological verbs was paired with either the agentBiasing or the Neutral context (agentBiasing-PsychV, Neutral-PsychV). These control target sentences were not paired with the constBiasing context because psychological verbs do not yield a constitutive reading (such that the subject is conceived as a subpart of the entity denoted by the complement). The critical verbs of the target sentence in the constBiasing-AspV condition appeared in the present-tense forms to facilitate the constitutive reading; for all other conditions the critical verbs appeared in the past-tense forms. Table 1 provides an example set of the critical conditions. ${ }^{2}$

We checked the frequencies of the critical verbs using the Corpus of Contemporary American English (COCA) for both their lemma forms and the exact forms as they appeared in the target sentences. To examine whether verb frequency differed by Verb Type, we performed a mixed-effects model analysis with the lmer function of the lme 4 package (Bates et al., 2015) in the R statistical package environment (R Core Team, 2015; RStudio Team, 2016). An effect of verb type was evaluated by contrasting a model with the fixed factor of verb type against a base model without it. Both models incorporated a random intercept for item-set (given that models with the random slope for verb type failed to converge). Analysis of lemma frequencies showed that the aspectual verbs were significantly more frequent than the control psychological verbs $\left(\operatorname{Mean}_{A s p V}=67283.2, \mathrm{SD}_{A s p V}=30510.73\right.$, $\operatorname{Mean}_{P \text { sych }}=43240, \quad \mathrm{SD}_{\text {PsychV }}=70293.29 ; \quad \chi^{2}(1)=$ $11.77, p<.001)$. The same difference in frequency was found with the exact forms appeared in the

\footnotetext{
${ }^{2}$ We note that the ambiguity examined here differs from that examined in Traxler et al. (2005)'s study. The latter tackles the ambiguity regarding the specific activity involving the subject as an agent and the complement as a theme. On the SIH, all kinds of activity fall within the eventive dimension and therefore the context manipulated in Traxler et al. is associated solely with the agentive reading along the eventive dimension. Indirectly supporting the SIH, Frisson and McElree (2008) also show that processing cost is not modulated by the number of activity interpretations associated with the target sentence containing underspecified meaning like "The teenager began the novel.". Instead, our study addresses the ambiguity among various dimensions, including not only the eventive dimension, which encompasses various activities, but also the spatial and informational dimensions etc.. As such, the specific activity in the agentive reading along the eventive dimension need not be made precise in order for the sentence to be interpreted.
} 
target sentences $\left(\mathrm{Mean}_{A s p V}=70653.59, \mathrm{SD}_{A s p V}\right.$ $=53930.98, \operatorname{Mean}_{\text {PsychV }}=12378.67, \mathrm{SD}_{\text {PsychV }}=$ $\left.13582.16 ; \chi^{2}(1)=59.96, p<.001\right)$. Previous studies have evidenced that higher lexical frequencies are associated with lesser processing costs (Inhoff and Rayner, 1986; Rayner and Duffy, 1986; Just and Carpenter, 1980; Kliegl et al., 2004; Rayner and Raney, 1996; Rayner et al., 1996). Therefore, an effect of frequency in our study would bias toward a finding of lesser processing cost for aspectual-verb sentences, which runs counter to our prediction that aspectual-verb sentences will be more costly than the control psych-verb sentences.

The stimuli were also submitted to an acceptability rating questionnaire $(n=29)$, in which the participants were asked to rate each passage consisting of a context sentence and a target sentence and then choose the plausible readings for the target sentence given the context. Results showed that the passages were within the acceptable range $>3.5$ in average (agentBiasing-AspV: Mean=3.84, SD=1.00; Neutral-AspV: Mean=3.59, $\mathrm{SD}=1.03 ; \quad$ constBiasing-AspV: $\quad$ Mean=3.69, $\mathrm{SD}=0.98 ; \quad$ agentBiasing-PsychV: $\quad$ Mean $=4.06$, $\mathrm{SD}=0.96$; Neutral-PsychV: Mean=3.84, $\mathrm{SD}=1.07$ ). Moreover, the target aspectual-verb sentences (e.g., Dave Brubeck started this CD) elicited an agentive reading (Dave Brubeck started listening to this CD) when following an agentiveBiasing context and elicited a constitutive reading (The song by Dave Brubeck was the first song on this CD) when following a constBiasing context. Following a neutral context, the aspectual-verb sentences gave rise to both agentive and constitutive readings with comparable selection rates (Lai and Piñango, 2017b, under review).

In addition, fifty filler items were introduced; half of them were grammatical and the other half were ungrammatical. Each grammatical/ungrammatical foil condition contained half aspectual verbs and half control verbs. Meanwhile, thirty of the foils involved the constBiasing context to counterbalance the context type across the stimuli. The whole set of experimental sentences amounted to 200 passages in total ( 30 sets of the 5 critical conditions plus 50 foil items). The stimuli were pseudo-randomized for each participant. To do so, we created four master scripts, splitting the stimuli into 30 blocks; each block contained 6 7 items of distinct condition and distinct item-set index. We then used these four master scripts to generate distinctive participant scripts randomly. The block order as well as the order of the items within each block differed in each participant script.

To ensure that the participants attend to the reading task, we inserted Yes/No comprehension questions after the target sentences for $1 / 3$ of the tri- als (68 out of 200 items). These questions were distributed across conditions, interspersed randomly throughout the experiment. The correct answers were half "Yes" and half "No." To avoid strategic reading, half of the comprehension questions probed the content of the context sentences and the other half probed the content of the target sentences.

\subsection{Participants}

Fifty-one native speakers of American English were recruited ( 36 females), all were between the ages of 18 30, with normal or corrected-to-normal vision and without reading disabilities by selfreport. The experiment protocol was approved by the Human Subjects Committee of Yale University; informed consent was obtained from each participant prior to the experiment. The participants received monetary compensation for participating the experiment.

\subsection{Apparatus $\mathcal{E}$ Procedures}

We recorded binocular eye-movements using an EyeLink 1000 Plus eye-tracker at a 500Hz sampling rate. The participants were instructed to place their heads on a chin and forehead rest, placed about 68 $\mathrm{cm}$ from the computer screen used for stimulus presentation. Sentences were displayed in a monospace font (Consolas), with about 3.39 characters per degree of visual angle. The context sentences were displayed on either one or two lines on the screen depending on length. Sentences were left-aligned with the left margin set to be 85 pixels from the edge of the screen. The target sentences fitted in one line, vertically centered on the screen and leftaligned with the same margin as for context sentences.

The experiment was run in three sub-sessions with short breaks in between. Each sub-session began with a screen presenting the task instruction, followed by the eye-tracking calibration with a series of 13 fixation targets across the display. The participants were instructed to sit in front of the display screen with their heads placed on the head-mount. Each trial began with a target dot (shown as a fake drift check) at the left-edge of the screen to signal the starting point of the context sentence. The participants, when ready, fixated on the target dot and pressed the spacebar, which brought up the context sentence. After finishing reading it, the participants pressed the spacebar, and then another fixation target dot (presented as a drift check) appeared at the left-edge of the screen. Similarly, the participants fixated on the dot and pressed spacebar to bring up the target sentence. When finishing reading it, they pressed the spacebar again.

For $1 / 3$ of the trials, a comprehension question was shown immediately after the target sentence, to 
Table 1: Sample stimuli

\begin{tabular}{l|l|l}
\hline Condition & Context sentence & Target sentence \\
\hline agentBiasing-AspV & $\begin{array}{l}\text { Musicians often record their pieces for } \\
\text { compilation or memorial albums. } \\
\text { Many musicians have music libraries that } \\
\text { contain tons of albums. }\end{array}$ & $\begin{array}{l}\text { Dave Brubeck started this CD of } \\
\text { classic Jazz hits. }\end{array}$ \\
\hline Neutral-AspV & $\begin{array}{l}\text { Kevin owns numerous CDs by different } \\
\text { Jazz musicians. }\end{array}$ & $\begin{array}{l}\text { Dave Brubeck starts this CD of classic } \\
\text { Jazz hits. }\end{array}$ \\
\hline constBiasing-AspV & $\begin{array}{l}\text { Musicians often record their pieces for } \\
\text { compilation or memorial albums. } \\
\text { agentBiasing-PsychV } \\
\text { Nany musicians have music libraries that } \\
\text { contain tons of albums. }\end{array}$ & $\begin{array}{l}\text { Dave Brubeck loved this CD of classic } \\
\text { Jazz hits. }\end{array}$ \\
\hline
\end{tabular}

which the participants answered by pressing the left arrow $(\leftarrow)$ for "Yes" or the left arrow $(\rightarrow)$ for "No." The response options were provided on the screen at the positions corresponding to the arrow keys on the keyboard (i.e. "Yes" on the left; "No" on the right) as a reminder. Once the response to the question was made, the screen presented a trial-interval page showing "Hit the spacebar for the next trial." For those trials without a comprehension question, the display screen proceeded to this transition page directly. Prior to the real session, a practice session with six trials was given to familiarize the participants with the experimental procedure.

\subsection{Data analysis}

Eye movements were recorded from both eyes, but data from the right eye was used for analysis by default. Four participants' right-eye data were unusable and so we analyzed their left-eye data. One participant whose fixations fell outside of the predetermined boundaries of the interest areas for more than $1 / 4$ of the trials was excluded from further data analysis. Fixations whose durations were shorter than 50ms were merged with the nearby fixations (Carpenter and Just, 1983; Katsika et al., 2012). Prior to the statistical analysis, we excluded trials using the following criteria: (a) target sentences containing more than three blinks $(2.946 \%)$, (b) target sentences with reading times longer than 10 seconds $(0.227 \%)$, (c) target sentences in which the first fixation did not fall within the region containing the subject noun phrase $(4.548 \%)$, and (d) trials which were not recorded properly due to technical errors $(1.307 \%)$. These combined excluded $9.029 \%$ of the critical trials in total.

The critical target sentences were segmented into five regions following the region definitions of Katsika et al. (2012)'s eye-tracking study; see Table 2. Region 1 contained the subject noun phrase; Region 2 contained the critical verb up to the word prior to the complement head noun; Region 3 included the complement head and the following word. Region 4 included the two words following Region 3; in cases whether both were function words, the region extended to the following word so that at least one of the word in the region was a content word. The remaining words of the sentence were assigned to Region 5. By the $\mathrm{SIH}$, our regions of interest were Region 2 that contained the critical verb, where the lexical-dimension functions of aspectual verbs are retrieved, Region 3 that contained the complement head, where the available dimension are construed from the complement's denotation, and Region 4, which was included to capture lasting effect associated with ambiguity resolution. Katsika et al. (2012) was the first study to dissociate aspectual verbs from psychological verbs and showed a processing difference between the two. Following the same regioning scheme used there allows for direct comparison of our results with theirs.

Four eye-movement variables were examined for each region of interest based on previous studies on processing underspecified meaning (e.g., Traxler et al., 2005; Katsika et al., 2012; Delogu et al., 2017). (1) First-pass duration sums all first-run fixations, including the first fixation in the region until the eyes leave it. This variable is intended to measure effects associated with the processing of lexical information (Rayner and Duffy, 1986; Rayner and Frazier, 1989; Starr and Rayner, 2001). (2) First-pass regression indicates whether the sequence of first-pass fixations in a region ends in a backward glance to an earlier region; this measurement is coded dichotomously as 0 or $1-1$ if the participant regresses back to an earlier region after the first fixation within the region and 0 otherwise. (3) Regression-path duration sums all fixation durations within a region, including the first fixation until the eyes exist the region. These variables are measured to examine processing difficulty associated with meaning composition, integration with information received by far, and disambiguation by context (Clifton et al., 2007; Rayner, 1998). (4) Regressions-in indicates whether the eyes enter a re- 
Table 2: Region definitions of the target sentences

\begin{tabular}{llllll}
\hline Condition & Reg. 1 & Reg. 2 & Reg. 3 & Reg. 4 & Reg. 5 \\
\hline AspV & Dave Brubeck & started this & CD of & classic Jazz & hits. \\
PsychV (Control) & $\ldots \ldots$ & loved this & $\ldots \ldots$ & $\ldots \ldots$ & $\ldots \ldots$ \\
\hline
\end{tabular}

gion from a latter region, coded dichotomously-1 if the region receives at least one regression from a later part of the sentence and 0 otherwise. This measurement is incorporated to examine where readers regress back to an earlier region when encountering processing difficulty. These variables were analyzed in the $\mathrm{R}$ statistical environment ( $\mathrm{R}$ Core Team, 2015; RStudio Team, 2016), using the lme 4 package (Bates et al., 2015). Continuous dependent variables (first-pass durations, regression-path durations) were analyzed by linear mixed-effects models whereas dichotomous dependent variables (firstpass regressions, regression-in) were analyzed by mixed-effects logistic regressions.

Two sets of analyses were performed to address two research questions. In Analysis 1, we examined the effect of Verb Type (AspV, PsychV), Context Type (agentBiasing, Neutral), and their interaction as a $2 \times 2$ design. An interaction effect was assessed by contrasting a full model that incorporated verb type and context type as two fixed factors as well as their interaction against the additive model with the interaction term dropped (Field et al., 2012; Winter, 2013). An effect of verb type was assessed by contrasting the additive model against a model with the fixed factor of verb type dropped. Likewise, an effect of context type was evaluated by contrasting the additive model against a model with the fixed factor of context type dropped. In Analysis 2, we examined the effect of the three context types (Neutral, agentBiasing, constBiasing) on the processing of aspectual-verb sentences only. An effect of context was evaluated by contrasting a model that contained a fixed factor of context type against a base model without it.

All models incorporated standardized region word-lengths as a covariate. We began with the maximal model with full random effect structure and simplified step-wise due to convergence failure. The final random effect structure of the models included random intercepts for participant and itemset as well as a random slope for word-length over participant yet with their correlation term removed. In addition, Bonferroni correction was carried out when evaluating effect significance, for which the critical $p$-value of .05 was divided by 12 to account for the 3 regions of interest and the 4 measurement variables, thresholding at $p=.0042$ (von der Malsburg and Angele, 2017).

\section{Results}

The accuracy of the comprehension questions achieved $92.79 \%$ on average, with all participants performing above $80 \%$, suggesting that the participants attended to the reading task. With respect to the eye-movement measurements, we reported the results for each set of analysis below.

Analysis 1, which crossed 2 verb types with 2 context types, showed a significant main effect of verb type in all three regions of interests. Specifically, Region 2 showed that aspectual-verb sentences engendered longer reading times (RTs) than the control-PsychV sentences in regression-path $d u$ rations $\left(\chi^{2}(1)=9.887, p<.002\right)$ and regression-in $\left(\chi^{2}(1)=19.683, p<.001\right)$. At Region 3, this verbtype effect was also found in first-pass regressions $\left(\chi^{2}(1)=31.855, p<.001\right)$. The same effect appeared at Region 4 as well in regression-path durations $\left(\chi^{2}(1)=8.378, p<.004\right)$. An opposite verb-type effect was shown at Region 3, such that psychological verbs induced longer RTs than aspectual verbs in first-pass durations $\left(\chi^{2}(1)=9.109, p<.003\right)$. On the other hand, Analysis 2, which compared the three context types for aspectual-verb sentences only, revealed no significant effect at any region. The mean reading times are summarized in Table 3 and visualized in Figure 2. The statistical results of the mixedeffect models showing the verb-type effect are provided in Appendix A.

Overall, results showed that aspectual-verb sentences incurred more processing cost than the control PsychV sentences in later measurements at all regions of interest. No effect of context type nor interaction was found in neither sets of analyses.

\section{Discussion}

The eye-movement results show that processing aspectual-verb sentences engendered more cost as compared to controls, replicating previous studies on this verb class (Katsika et al., 2012; Lai et al., 2017a; Zarcone et al., 2017). The cost revealed itself in the regions containing the critical verb, the complement head, and a few words following it. The pattern is consistent with the third outcome outlined in Section 1.3: the cost associated with the aspectual-verb composition is present irrespective of context and lingers after the complement is retrieved. In what follows, we explicate the processing 
Table 3: Mean and standard errors of the eye-movement measurements per region of interest

\begin{tabular}{llllll}
\hline Condition & Region & $\begin{array}{l}\text { First-pass duration } \\
(\mathrm{se})\end{array}$ & $\begin{array}{l}\text { First-pass re- } \\
\text { gression (se) }\end{array}$ & $\begin{array}{l}\text { Regression-path } \\
\text { duration (se) }\end{array}$ & $\begin{array}{l}\text { Regression-in } \\
(\mathrm{se})\end{array}$ \\
\hline Neutral-AspV & & $339.74(5.82)$ & $0.06(0.01)$ & $386.92(7.83)$ & $0.52(0.01)$ \\
agentBiasing-AspV & & $336.69(5.69)$ & $0.07(0.01)$ & $379.33(7.31)$ & $0.53(0.01)$ \\
ConstBiasing-AspV & \multirow{2}{*}{ Reg.2 } & $340.90(6.01)$ & $0.09(0.01)$ & $397.48(7.84)$ & $0.51(0.01)$ \\
Neutral-PsychV & & $344.07(5.69)$ & $0.06(0.01)$ & $378.23(6.87)$ & $0.46(0.01)$ \\
agentBiasing-PsychV & & $338.04(5.41)$ & $0.05(0.01)$ & $372.39(7.31)$ & $0.47(0.01)$ \\
\hline Neutral-AspV & & $319.72(5.79)$ & $0.25(0.01)$ & $466.18(10.68)$ & $0.40(0.01)$ \\
agentBiasing-AspV & & $319.72(5.41)$ & $0.26(0.01)$ & $471.76(10.43)$ & $0.40(0.01)$ \\
ConstBiasing-AspV & Reg.3 & $318.98(5.81)$ & $0.24(0.01)$ & $481.18(12.03)$ & $0.39(0.01)$ \\
Neutral-PsychV & & $345.19(6.53)$ & $0.19(0.01)$ & $460.52(11.11)$ & $0.37(0.01)$ \\
agentBiasing-PsychV & & $328.42(5.64)$ & $0.19(0.01)$ & $444.87(9.81)$ & $0.40(0.01)$ \\
\hline Neutral-AspV & & $408.45(8.22)$ & $0.44(0.01)$ & $855.74(20.86)$ & $0.25(0.01)$ \\
agentBiasing-AspV & & $430.36(9.63)$ & $0.42(0.01)$ & $888.68(22.25)$ & $0.23(0.01)$ \\
ConstBiasing-AspV & Reg.4 & $415.69(8.83)$ & $0.41(0.01)$ & $844.53(21.88)$ & $0.26(0.01)$ \\
Neutral-PsychV & & $435.59(8.92)$ & $0.40(0.01)$ & $818.35(20.21)$ & $0.25(0.01)$ \\
agentBiasing-PsychV & & $409.35(8.04)$ & $0.41(0.01)$ & $817.77(19.54)$ & $0.25(0.01)$ \\
\hline
\end{tabular}
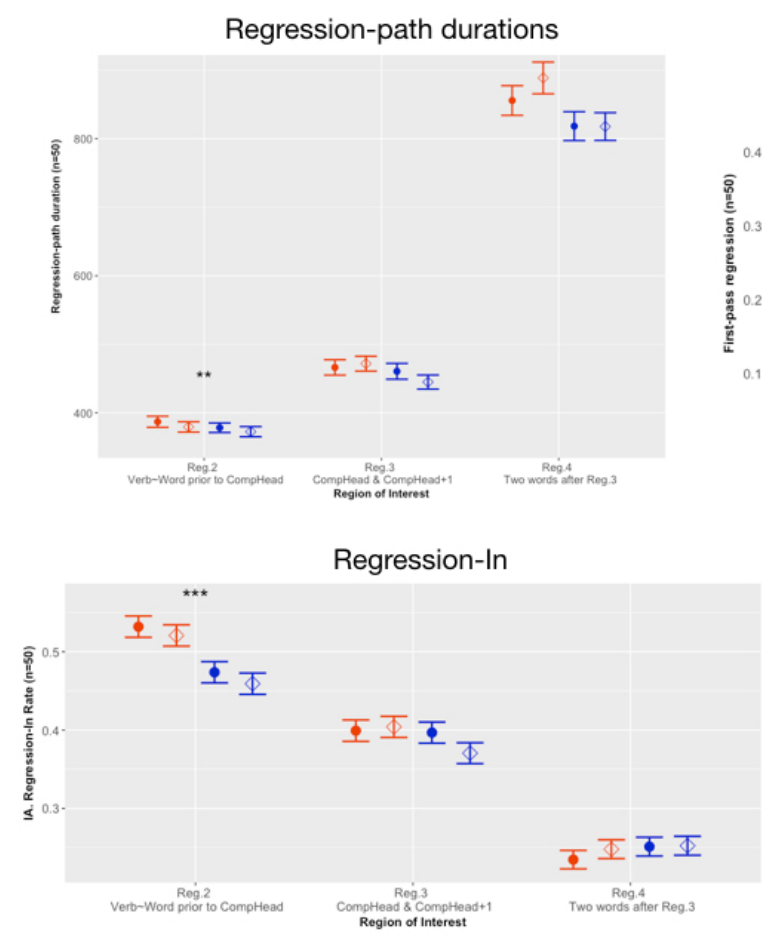

First-pass regression

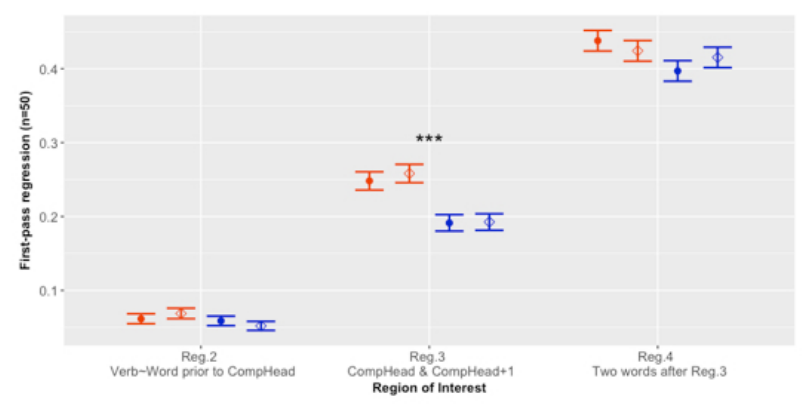

First-pass durations

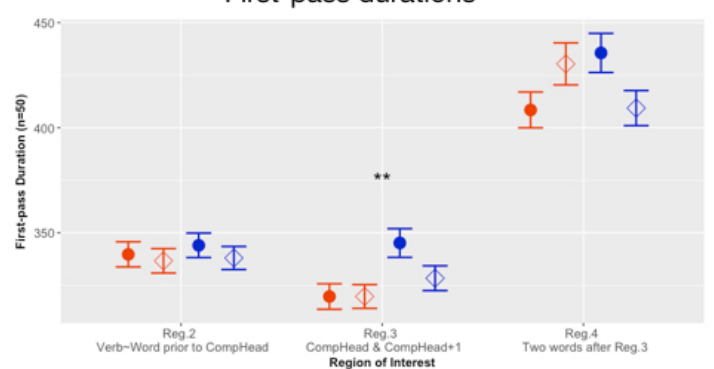

VerbType AspV PsychV ContextType Neutral $\bigcirc$ agentBiasing

Figure 2: Eye-movement results 
load associated with aspectual-verb composition in Section 4.1, followed by the discussion on the lack of immediate context attenuation in Section 4.2.

\subsection{The processing cost of aspectual-verb compo- sition (> controls)}

We interpret the observation that the cost is observed in relatively later measurements, i.e. regression-path durations and regressionin at Reg.2, first-pass regressions at Reg.3, and regression-path durations at Reg.4, as directly reflective of readers' construal of dimension-specific structured individuals, including the maintenance of these alternative representations once construed. As predicted, these processes are invoked by aspectualverb composition and not the control counterparts. This verb-type effect took place irrespective of context bias, suggesting that aspectual-verb's dimension composition is context-independent.

We note that semantic composition necessarily involves lexical retrieval of the verb's functions and the complement denotation. Lexical retrieval has been demonstrated to be exhaustive and contextimpenetrable (Duffy et al., 1988; Elston-Güttler and Friederici, 2005; Klepousniotou et al., 2012; Love and Swinney, 1996; Shapiro et al., 1989; Shetreet et al., 2016; Swinney, 1979; Swinney et al., 2000). For example, Shapiro et al. (1989) show that verbs encoding more argument structure options (e.g., dative verbs such as send) elicit longer response times than verbs with fewer options (e.g., transitive verbs such as fix) in a cross-modal lexical decision experiment. This is the case regardless of context. More recently, Shetreet et al. (2016) report that verbs with multiple semantic/subcategorization frames (e.g, remember, which takes a theme/NP or a proposition/embedded clause) increase activity in left posterior superior temporal gyrus than verbs with only one frame (e.g., punish, which takes only a theme/NP) in their fMRI study. In our case, aspectual verbs do allow multiple thematic structures associated with different readings, as shown in (5) in Section 1.3. These findings indicate that all lexical information of verb are exhaustively activated during real-time comprehension.

Of relevant note, a recent study shows that the comprehension of aspectual-verb sentences with either animate-denoting or inanimate-denoting subjects (e.g., The prologue / noblewoman began the novel.) invokes comparably longer reading times and increases activity in left middle temporal gyrus similarly (Lai et al., 2016). This is argued to result from the exhaustive retrieval of the lexical functions encoded in aspectual verbs (e.g., $f_{\text {space }}, f_{\text {info }}$, $\left.f_{\text {event }}, f_{\text {time }}\right)$, which takes place irrespective of the subject. More specifically, suppose that without supporting context, aspectual-verb sentences with inanimate-denoting subjects (e.g., The prologue began the novel.) yield the constitutive reading primarily while those with animate-denoting subjects (e.g., The noblewoman began the novel.) license both agentive and constitutive readings. Results of Lai et al. (2016) suggest that all possible dimension representations are construed independent of sentential context, as a consequence of the exhaustive lexical retrieval of the dimension-functions, consistent with literature (Duffy et al., 1988; Onifer and Swinney, 1981; Rayner and Duffy, 1986; Seidenberg et al., 1982; Tanenhaus et al., 1990). The composition of exhaustively activated lexical representations of an aspectual verb and its complement gives rise to multiple semantic representations, leading to dimension ambiguity at the sentence level consequently. With this consideration, we take the similar processing profiles of the three aspectual-verb conditions under different contexts to reflect the construal and maintenance of multiple dimension-specific structured individuals, invoked by meaning implementation of aspectual verbs, while the exact dimension representation is left underspecified.

\subsection{No immediate effect of biasing context}

In addition to the main effect of verb type (aspectual-verbs $>$ controls), we did not find an immediate effect of context type, i.e. agentBiasing$\mathrm{AspV} \cong$ constBiasing-AspV $\cong \mathrm{Neutral}-\mathrm{AspV}$. Processing aspectual-verb sentences in a biasing context, either towards the agentive reading or the constitutive reading, is as costly as doing so in a neutral context that is compatible with both readings.

On the other hand, Lai and Piñango (2017b; under review) demonstrate-with the same stimuli-that comprehenders arrived at the reading(s) biased by context eventually when they were asked to give an interpretation of the sentence in an offline questionnaire study. The participants obtained the agentive reading for the target aspectual-verb sentences under the agentive-biasing context while they obtained the constitutive reading under the constitutive-biasing context. This evidence suggests that context does modulate ultimate sentence interpretation. The difference in the effect of context between the online and offline measurements points to a timing issue, suggesting that context effect is largely delayed and may only be observable when the processor is forced to make a choice obligatorily (Frazier and Rayner, 1990; Frazier, 1999).

Given that the effect of context does not emerge intra-sententially online in the current study but appears extra-sententially offline in the study of Lai and Piñango (2017b), we suggest that the role of context in processing underspecified meaning is to privilege the appropriate reading(s) without eliminating the alternatives (Figure 3). If the processor 
pre-selects the representation favored by prior context and eliminates other alternatives right away, the cost for the target aspectual-verb sentences in the biasing contexts should be attenuated, contrary to the results. The lack of immediate context modulation suggests that whatever privileging properties context has, it does not contribute to the decay of the alternative underspecified readings of sentences. This is so because the alternative dimensions are compatible with the complement's conceptual representation (while the dimension representations are mutually exclusive to each other like the Necker cube). For example, the situation in which one reads the book does not necessarily exclude the conception of the book as consisting of a series of chapters. As dimensions are perspectives of looking at the same structured individual denoted by the complement, there is no strong reason to actively eliminate one or another; all of them may hold during comprehension, in particular since our comprehension task did not ask the participants to choose among available readings.

We take these findings to indicate that contextual cues have little impact on the initial composition of aspectual-verb sentence meaning during realtime processing though it factors into the final interpretation at a later time point. This interpretation is in line with previous studies on the processing of verbs with multiple senses (Frazier and Rayner, 1990; Frisson and Pickering, 1999; Frisson, 2009; Frisson and Pickering, 2001; Pickering and Frisson, 2001). In an eye-movement study, Frazier and Rayner (1990) compare nouns with multiple meanings (e.g., the concrete vs. abstract meaning of bug) with those with multiple senses (e.g., the concrete vs. abstract sense of book) in sentential context with disambiguating information. They found an effect of context attenuation for ambiguous words with multiple meanings but not for words with multiple senses. Accordingly, the authors argue for the Partial specification hypothesis; it claims that the various senses of a word share common semantic features, according to which the processor which will assign as an initial interpretation for the target sentence. The exact sense may later be specified when more information is obtained, at a cost much smaller than resolving the ambiguity for words with multiple unrelated meanings. Semantic commitment is only partial, especially when nothing in context requires full meaning specification.

Pickering and Frisson (2001) show that while context facilitation appears relatively quickly for verbs with multiple unrelated meanings (e.g., "rule the country/line"), it is much delayed for verbs with multiple related senses (e.g., "disarm every rebel/critic"), similar to nouns with multiple senses (Frisson and Pickering, 1999), and only observable at the end-of line region with later measurements. The authors suggest that word senses are not necessarily in competition with each other; context plays its role not in picking out one particular sense among the alternatives but rather in constraining the interpretation. The processor activates an underspecified meaning and may later home-in on a more specified meaning that is specific to the given context. The delayed cost is attributed to late meaning integration, manifested as a sentence wrap-up effect. The delay in semantic commitment is considered advantageous because in a particular sentence the interpretation of verbs is highly dependent on the arguments they combine with (Pickering and Frisson, 2001, p.557), and the processor is therefore expected to wait until no further useful information is provided. This is consistent with results from Braze et al. (2002), whose eye-tracking experiment shows that sentences with pragmatic violation incurred more regressions in the sentence-final region, suggesting that the processor delays full commitment and defers meaning integration towards the end of sentences.

We suggest that dimension-specific structured individuals construed through aspectual-verb composition behave like word senses. Dimension ambiguity is a kind of sense ambiguity in that the various dimension representations are compatible with the complement denotation and share common semantic features (parthood relations as a selectional constraint). What both cases have in common is that the interpretations (senses for nouns/verbs, dimensions for the aspectual class) are not necessarily in competition with each other, and need not to be eliminated early on, hence the delayed contextual resolution. Under our proposed approach, meaning ambiguity in the current case results from a combinatorial process, engendered by the aspectual verb, in composition with its complement. We argue that such compositional ambiguity resolution is more demanding than ambiguity that does not emerge compositionally but is characterizable strictly as lexically encoded (i.e. lexical polysemy). The compositional nature of this ambiguity leads to a further delayed context impact. Such impact was not visible within the regions examined but is observable extra-sententially in the form of increased sentence acceptability (Lai and Piñango, 2017b).

The lack of context effect during real-time processing along with the delayed semantic commitment for underspecified meaning is consistent with a kind of Good-Enough approach to processing (Ferreira et al., 2002; Ferreira and Patson, 2007; Sanford and Sturt, 2002). It claims that sentence comprehension is not always complete and flawless, but just "good enough" or in our case "specific enough" to cope with the task at hand, which in our case 
Figure 3: Context constraining

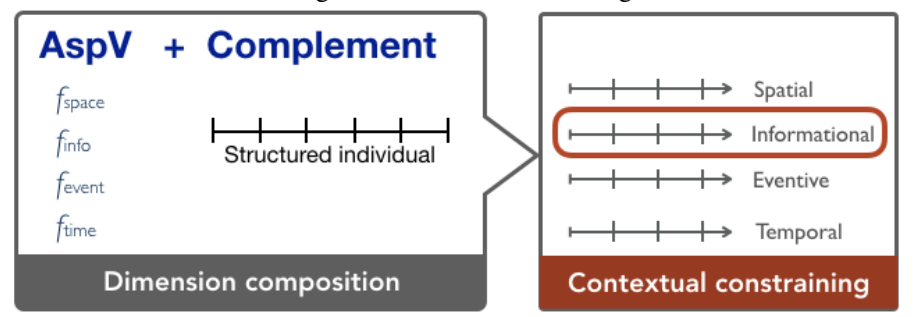

it would be the construal of a viable semantic representation. Ferreira and Bailey (2004) show that the comprehension system does not eliminate errors during speech from its representation of the utterance; the incorrect representation lingers and may even be allowed to interfere with global comprehension. Along similar lines, Frazier et al. (1999) report that people do not commit to a distributed or non-distributed reading of sentences like "Mary and John saved \$100." and only later do they specify the interpretation if such precisification is required. With respect to contextual impact, Tunstall (1998) shows that sentences with scope ambiguity are processed alike in biasing contexts toward either the preferred or dis-preferred reading. A similar view is taken by the underspecification account to figurative language processing (e.g., Frisson and Pickering, 1999; Pickering and Frisson, 2001). For instance, Frisson and Pickering (1999) found comparable processing cost for the figurative as well as the literal meaning of metonymies. They argue that a relatively coarse representation compatible with both literal and figurative senses is activated initially, and the processor may subsequently specify an exact interpretation, if required.

From this constraint-based perspective, contextual constraints can be implemented for monitoring and adjusting the common ground, the information mutually believed by conversation participants in discourse; it serves as the domain of interpretation (Clark et al., 1991; Hanna et al., 2003). In the beginning of the conversation, the shared common ground is limited, and the search domain for identifying the speaker's meaning is broad. As sentences proceed and more information is provided, the shared common ground is enlarged and the interpretation domain narrowed down incrementally. In other words, what context does is to constrain the shared common ground for the comprehender to identify the intended meaning, rather than singling out one particular meaning out of multiple alternatives. Interestingly, Horton and Keysar (1996) show that the effect of context integration for tailoring the shared common ground is observable only when people are not under time pressure while unobservable under time pressure, supporting that context constraining is in- corporated at a later stage as a monitoring and adjusting device during comprehension.

Taken together, these findings suggest that the semantic processor does not commit to a specific interpretation among the alternatives (as in the cases of multiple related senses), unless forced to do so in order to avoid inconsistency (as in the cases of multiple unrelated meanings). The effect of context is delayed and may not be observed during realtime processing under time pressure although it is observable in offline interpretation-when comprehenders are forced to make a choice. Furthermore, dimension ambiguity resolution by contextual integration takes place in the form of privileging some dimension-specific representation(s) without eliminating other alternatives completely. The system of processing underspecified meaning revealed here is consistent with a good-enough processing system for semantic composition.

\section{Conclusion}

In this study, we test sentences with underspecified meaning under biasing vs. neutral context using aspectual verbs. Results from eye-movements show that, when syntactic components are factored out, sentences which involve multiple semantic representations, and therefore ambiguity, are more costly than the unambiguous counterparts-regardless of contextual bias during real-time comprehension. The processing cost is taken to result from exhaustive retrieval of the lexical functions encoded in aspectual-verbs as well as the construal and the maintenance of multiple dimension representations. With respect to the time-course of the context modulation, we suggest that its constraining impact is largely delayed during processing underspecified meaning, detectable extra-sententially only when the processor is forced to make a choice. Ambiguity resolution in this case is implemented by privileging the appropriate semantic representation(s) without eliminating the alternatives completely during realtime comprehension. The pattern is consistent with a lexically-driven, contextually-constraining system for processing underspecified sentence meaning. 


\section{Acknowledgement}

This research was funded by NSF-BCS grant BCS-0643266 to Maria Mercedes Piñango, and NSF-INSPIRE Grant CCF-1248100 to Maria Mercedes Piñango, Ashwini Deo, Todd Constable, and Mokshay Madiman. David Braze's effort on this project was supported by NIH grant R01 HD071988.

\section{References}

Bates, D., Mächler, M., Bolker, B., Walker, S., 2015. Fitting linear mixed-effects models using lme4. Journal of Statistical Software 67 (1), 1-48.

Braze, D., Shankweiler, D., Ni, W., Palumbo, L. C., 2002. Readers' eye movements distinguish anomalies of form and content. Journal of psycholinguistic research 31 (1), 25-44.

Carpenter, P. A., Just, M. A., 1983. What your eyes do while your mind is reading. In: Eye movements in reading. Elsevier, pp. 275-307.

Clark, H. H., Brennan, S. E., et al., 1991. Grounding in communication. Perspectives on socially shared cognition 13 (1991), 127-149.

Clifton, C., Staub, A., Rayner, K., 2007. Eye movements in reading words and sentences. In: Eye Movements. Elsevier, pp. 341-371.

Delogu, F., Crocker, M. W., Drenhaus, H., 2017. Teasing apart coercion and surprisal: Evidence from eye-movements and erps. Cognition 161, 46-59.

DiNardo, L., 2015. Competing analyses of complement coercion: new evidence from behavioral and electropsychophysiological methods. Senior thesis.

Duffy, S. A., Morris, R. K., Rayner, K., 1988. Lexical ambiguity and fixation times in reading. Journal of memory and language 27 (4), 429-446.

Elston-Güttler, K. E., Friederici, A. D., 2005. Native and 12 processing of homonyms in sentential context. Journal of Memory and Language 52 (2), 256-283.

Ferreira, F., Bailey, K. G., 2004. Disfluencies and human language comprehension. Trends in cognitive sciences 8 (5), 231-237.

Ferreira, F., Bailey, K. G., Ferraro, V., 2002. Good-enough representations in language comprehension. Current directions in psychological science 11 (1), 11-15.

Ferreira, F., Patson, N. D., 2007. The 'good enough'approach to language comprehension. Language and Linguistics Compass $1(1-2), 71-83$.

Field, A., Miles, J., Field, Z., 2012. Discovering statistics using R. Sage publications.

Frazier, L., 1999. On sentence interpretation. Vol. 22. Springer Science \& Business Media.

Frazier, L., Pacht, J. M., Rayner, K., 1999. Taking on semantic commitments, ii: collective versus distributive readings. Cognition 70 (1), 87-104

Frazier, L., Rayner, K., 1990. Taking on semantic commitments: Processing multiple meanings vs. multiple senses. Journal of memory and language 29 (2), 181.

Frisson, S., 2009. Semantic underspecification in language processing. Language and Linguistics Compass 3 (1), 111-127.

Frisson, S., McElree, B., 2008. Complement coercion is not modulated by competition: Evidence from eye movements. Journal of Experimental Psychology: Learning, Memory, and Cognition 34 (1), 1

Frisson, S., Pickering, M. J., 1999. The processing of metonymy: evidence from eye movements. Journal of Experimental Psychology: Learning, Memory, and Cognition 25 (6), 1366.

Frisson, S., Pickering, M. J., 2001. Obtaining a figurative interpretation of a word: Support for underspecification. Metaphor and Symbol 16 (3-4), 149-171.
Hanna, J. E., Tanenhaus, M. K., Trueswell, J. C., 2003. The effects of common ground and perspective on domains of referential interpretation. Journal of Memory and Language 49 (1), 43-61.

Horton, W. S., Keysar, B., 1996. When do speakers take into account common ground? Cognition 59 (1), 91-117.

Husband, E. M., Kelly, L. A., Zhu, D. C., 2011. Using complement coercion to understand the neural basis of semantic composition: Evidence from an fmri study. Journal of Cognitive Neuroscience 23 (11), 3254-3266.

Inhoff, A. W., Rayner, K., 1986. Parafoveal word processing during eye fixations in reading: Effects of word frequency. Perception \& psychophysics 40 (6), 431-439.

Jackendoff, R., 1997. The architecture of the language faculty. MIT Press.

Just, M. A., Carpenter, P. A., 1980. A theory of reading: From eye fixations to comprehension. Psychological review 87 (4), 329.

Katsika, A., Braze, D., Deo, A., Piñango, M. M., 2012. Complement coercion: Distinguishing between type-shifting and pragmatic inferencing. The mental lexicon 7 (1), 58-76.

Klepousniotou, E., Pike, G. B., Steinhauer, K, Gracco, V., 2012 Not all ambiguous words are created equal: An eeg investigation of homonymy and polysemy. Brain and language 123 (1), 11-21.

Kliegl, R., Grabner, E., Rolfs, M., Engbert, R., 2004. Length, frequency, and predictability effects of words on eye movements in reading. European Journal of Cognitive Psychology 16 (12), 262-284.

Lai, Y.-Y., Lacadie, C., Constable, T., Deo, A., Piñango, M. M., 2016. The structured individual hypothesis for processing aspectual verbs. In: Proceedings of Berkeley Linguistics Society 42. pp. 135-152.

Lai, Y.-Y., Lacadie, C., Constable, T., Deo, A., Piñango, M. M., 2017a. Complement coercion as the processing of aspectual verbs: evidence from self-paced reading and fmri. In: Compositionality and Concepts in Linguistics and Psychology. Springer, pp. 191-222.

Lai, Y.-Y., Piñango, M. M., 2017b. Comprehending underspecified meaning: resolving ambiguity by contextual and conceptual search. In: Conference handbook of The 19th Annual International conference of the Japanese Society for Language Sciences. pp. 134-137.

Lai, Y.-Y., Piñango, M. M., under review. Context modulates the interpretation of underspecified meaning. Language and Cognition.

Love, T., Swinney, D., 1996. Coreference processing and levels of analysis in object-relative constructions; demonstration of antecedent reactivation with the cross-modal priming paradigm. Journal of Psycholinguistic Research 25 (1), 5-24.

McElree, B., Traxler, M. J., Pickering, M. J., Seely, R. E., Jackendoff, R., 2001. Reading time evidence for enriched composition. Cognition 78 (1), B17-B25.

Onifer, W., Swinney, D. A., 1981. Accessing lexical ambiguities during sentence comprehension: Effects of frequency of meaning and contextual bias. Memory \& Cognition 9 (3), 225-236.

Pickering, M. J., Frisson, S., 2001. Processing ambiguous verbs: Evidence from eye movements. Journal of Experimental Psychology: Learning, Memory, and Cognition 27 (2), 556.

Pickering, M. J., McElree, B., Traxler, M. J., 2005. The difficulty of coercion: A response to de almeida. Brain and Language $93(1), 1-9$.

Piñango, M. M., Deo, A., 2015. Reanalyzing the complement coercion effect through a generalized lexical semantics for aspectual verbs. Journal of Semantics 33 (2), 359-408.

Pustejovsky, J., 1995. The generative lexicon. MIT Press, Cambridge, MA.

R Core Team, 2015. R: A Language and Environment for Statistical Computing. R Foundation for Statistical Computing, Vienna, Austria.

URL https://www.R-project.org 
Rayner, K., 1998. Eye movements in reading and information processing: 20 years of research. Psychological bulletin 124 (3), 372.

Rayner, K., Duffy, S. A., 1986. Lexical complexity and fixation times in reading: Effects of word frequency, verb complexity, and lexical ambiguity. Memory \& cognition 14 (3), 191-201.

Rayner, K., Frazier, L., 1989. Selection mechanisms in reading lexically ambiguous words. Journal of Experimental Psychology: Learning, Memory, and Cognition 15 (5), 779.

Rayner, K., Raney, G. E., 1996. Eye movement control in reading and visual search: Effects of word frequency. Psychonomic Bulletin \& Review 3 (2), 245-248.

Rayner, K., Sereno, S., Raney, G., 11 1996. Eye movement control in reading: A comparison of two types of models. Journal of experimental psychology. Human perception and performance 22, 1188-200.

RStudio Team, 2016. RStudio: Integrated Development Environment for R. RStudio, Inc., Boston, MA.

URL http: //www.rstudio.com/

Sanford, A. J., Sturt, P., 2002. Depth of processing in language comprehension: Not noticing the evidence. Trends in cognitive sciences 6 (9), 382-386.

Seidenberg, M. S., Tanenhaus, M. K., Leiman, J. M., Bienkowski, M., 1982. Automatic access of the meanings of ambiguous words in context: Some limitations of knowledge-based processing. Cognitive psychology 14 (4), 489-537.

Shapiro, L. P., Zurif, E. B., Grimshaw, J., 1989. Verb processing during sentence comprehension: Contextual impenetrability. Journal of psycholinguistic research 18 (2), 223-243.

Shetreet, E., Linzen, T., Friedmann, N., 2016. Against all odds: exhaustive activation in lexical access of verb complementation options. Language, Cognition and Neuroscience 31 (9), 1206-1214.

Starr, M. S., Rayner, K., 2001. Eye movements during read- ing: Some current controversies. Trends in Cognitive Sciences 5 (4), 156-163.

Swinney, D., Prather, P., Love, T., 2000. The time-course of lexical access and the role of context: Converging evidence from normal and aphasic processing. In: Language and the brain. Elsevier, pp. 273-292

Swinney, D. A., 1979. Lexical access during sentence comprehension:(re) consideration of context effects. Journal of verbal learning and verbal behavior 18 (6), 645-659.

Tanenhaus, M. K., Garnsey, S. M., Boland, J., 1990. Combinatory lexical information and language comprehension. In: Altmann, G. T. M. (Ed.), ACL-MIT Press series in natural language processing. Cognitive models of speech processing: Psycholinguistic and computational perspectives. Cambridge, MA, US: The MIT Press, pp. pp. 383-408.

Traxler, M. J., McElree, B., Williams, R. S., Pickering, M. J., 2005. Context effects in coercion: Evidence from eye movements. Journal of Memory and Language 53 (1), 1-25.

Traxler, M. J., Pickering, M. J., McElree, B., 2002. Coercion in sentence processing: Evidence from eye-movements and selfpaced reading. Journal of Memory and Language 47 (4), 530547.

Tunstall, S. L., 1998. The interpretation of quantifiers: semantics \& processing. Ph.D. thesis, University of Massachusetts at Amherst.

von der Malsburg, T., Angele, B., 2017. False positives and other statistical errors in standard analyses of eye movements in reading. Journal of memory and language 94, 119-133.

Winter, B., 2013. Linear models and linear mixed effects models in $r$ with linguistic applications. arXiv preprint arXiv: 1308.5499

Zarcone, A., McRae, K., Lenci, A., Padó, S., 2017. Complement coercion: The joint effects of type and typicality. Frontiers in psychology 8, 1987.

\section{Appendix A. Statistical results of mixed-effect models on reading times}

\begin{tabular}{lllll}
\hline Region & First-pass duration & First-pass regression & Regression-path duration & Regression-in \\
\hline Reg. 2 & $\chi^{2}(1)=1.80, p=0.18$ & $\chi^{2}(1)=2.99, p=0.084$ & $\begin{array}{l}\text { AspV }>\text { Control: } \\
\chi^{2}(1)=9.887, p<.002\end{array}$ & $\begin{array}{l}\text { AspV }>\text { Control: } \\
\chi^{2}(1)=19.683, p<.001\end{array}$ \\
\hline Reg. 3 & $\begin{array}{l}\text { Control }<\mathrm{AspV}: \\
\chi^{2}(1)=9.109, p<.003\end{array}$ & $\begin{array}{l}\text { AspV }>\text { Control: } \\
\chi^{2}(1)=31.855, p<.001\end{array}$ & $\chi^{2}(1)=3.89, p=.049$ & $\chi^{2}(1)=2.18, p=.140$ \\
\hline Reg. 4 & $\chi^{2}(1)=0.53, p<.465$ & $\chi^{2}(1)=4.18, p=.041$ & $\begin{array}{l}\text { AspV }>\text { Control: } \\
\chi^{2}(1)=8.378, p<.004\end{array}$ & $\chi^{2}(1)=0.79, p=.374$ \\
\hline
\end{tabular}

